\title{
Explication et prévision des cinétiques de dégradation en Rusitec des matières azotées végétales à partir d'un fractionnement des parois
}

\author{
G Blanchart, B Vignon
}

INRA, Laboratoire de la chaire de zootechnie de I'ENSAIA, 2 avenue de la Forêt-de-Haye, 54500 Vandouvre-lès-Nancy, France

(Reçu le 24 octobre 1989; accepté le 25 avril 1990)

\begin{abstract}
Résumé - Les relations entre la solubilité de l'azote dans les détergents de Van Soest et la dégradation de l'azote in vitro ont été étudiées sur 11 aliments à partir de mesures de dégradabilité en rumen artificiel à flux semi-continu de type Rusitec. Les cinétiques de disparition de l'azote en Rusitec, et spécialement les taux de disparition maximale, sont assez proches des valeurs calculées à partir des données de la bibliographie obtenues in situ par la méthode des sachets. Dans le modèle exponentiel, utilisé pour décrire la dégradation de l'azote, la dégradation maximale $(a+b)$ est corrélée négativement à la proportion de l'azote insoluble dans le détergent acide de Van Soest (ADIN/N total). Pour les aliments testés, la fraction indégradable correspond à une quantité équivalente à environ $76 \%$ de la fraction $A D I N$. La dégradation instantanée (a) est, pour sa part, mieux expliquée par la proportion d'azote insoluble dans le détergent neutre de Van Soest (NDIN/N total) que par la solubilité de l'azote dans une solution saline. La vitesse de disparition (c) de la fraction lentement dégradable est inversement liée à la proportion de la fraction ADF dans les parois totales (NDF), qui témoigne de leur résistance à la dégradation. Ce type de fractionnement pourrait être utilisé pour prévoir la valeur azotée des aliments pour ruminants.
\end{abstract}

dégradation / azote / Rusitec / rumen / détergent

Summary - Utllization of a cell wall fractionation to explain and predict the kinetics of plant protein degradation in the rumen (Rusitec). The relation between nitrogen solubility in Van Soest detergents and in vitro nitrogen degradation was studied on 11 substrates with degradation process calculations in digestive trials and an antificial semi-continuous flow (Rusitec) rumen. The kinetics of plant protein degradation in Rusitec, especially the maximum degradation values, were rather similar to those calculated from literature data obtained in situ. In the exponential model used to describe the protein degradation, the maximum disappearance $(\mathrm{a}+\mathrm{b})$ was negatively correlated with the percentage of acid detergent insoluble nitrogen in the Van Soest (ADIN/Total N). In the foods tested, the non degradable fraction corresponds to a quantity which is equivalent to $\approx 76 \%$ of the ADIN fraction. The instantaneously degraded nitrogen (a) was rather well explained by the percentage of neutral detergent insoluble nitrogen in the Van Soest (NDIN/Total N). The rate of disapperance (c) of the slowly degraded fraction was inversely correlated with the proportion of ADF in the NDF, which demonstrates the latter's resistance to degradation. This type of fractionation could be used in predicting nitrogenous value of feed stuffs for ruminants. 


\section{INTRODUCTION}

La caractérisation de la valeur azotée des aliments prend le plus souvent en compte la notion de dégradabilité de l'azote et parfois, comme dans le système des PDI, celle de dégradation théorique, combinaison de la cinétique de dégradation et du temps de séjour dans le rumen (Jarrige, 1988).

La dégradation de l'azote dans le rumen est fonction de 3 catégories de paramètres :

- la dégradabilité de l'azote;

- les activités des micro-organismes du rumen, qui caractérisent leur potentiel de dégradation des substrats alimentaires;

- la durée pendant laquelle les matières azotées sont soumises aux activités des micro-organismes, c'est-à-dire leur temps de séjour dans le rumen.

La dégradabilité de l'azote est une caractéristique des aliments. Elle dépend, dans une large mesure, de la nature des matières azotées, qui détermine leurs propriétés physico-chimiques. À ce titre, la solubilité des protéines est l'un des principaux facteurs déterminant leur dégradabilité (Nocek et al, 1983; Cotta et Hespell, 1986). L'abondance des liaisons disulfures, qui peuvent assurer le maintient de la structure de la protéine, constitue cependant un frein aux activités enzymatiques (Mahadevan et al, 1980; Nugent et al, 1983). Enfin, certaines fractions de la matière organique peuvent protéger les protéines végétales des attaques microbiennes : les fractions hémicellulosique et cellulosique (Ørskov, 1982), les tannins (Zelter et al, 1970; Driedger et Hatfield, 1972) et la lignine (Hartnell et Satter, 1978; Thomas et al, 1979) sont celles qui ont été le plus étudiées. Les effets de l'ensemble de ces facteurs pourraient être évalués par la résistance à la solubilisation dans les détergents de Van Soest (Van Soest et Wine, 1967). Krishnamoorthy et al (1982a et b) ont ainsi considéré la fraction azotée insoluble dans le détergent neutre comme une estimation de la fraction lentement dégradable, et la fraction insoluble dans le détergent acide comme complètement inutilisable.

Les essais rapportés ici ont comme objectif de caractériser les relations existant entre la composition chimique des aliments, et spécialement leur composition azotée, et la dégradabilité de l'azote. Ces relations pourraient être utilisées pour proposer une technique de laboratoire simple pour prévoir la dégradabilité de l'azote dans le rumen.

\section{MATÉRIEL ET MÉTHODES}

L'appareil utilisé est un fermenteur artificiel à flux semi-continu du type du Rusitec proposé par Czerkawski et Breckenridge en 1977 et adapté par Blanchart et Vignon (1984), qui permet de travailler sur des quantités de produits (aliments, résidus de fermentation et contenus liquides) 2,5 fois plus importantes que dans le modèle original.

Les sachets destinés à recevoir les échantillons d'aliment sont fabriqués par thermosoudure en tissu de nylon de porosité $50 \mu \mathrm{m}$. Leurs dimensions $(15 \times 9 \mathrm{~cm})$ et la quantité d'aliment qui y est placée ( $14 \mathrm{~g}$ de matière sèche) établit le rapport "quantité de matière sèche/surface d'échange" à environ $52 \mathrm{mg} / \mathrm{cm}^{2}$. Cette valeur est plus élevée que celles rapportées le plus souvent dans la bibliographie pour la méthode des sachets, mais proche de celle habituellement proposée pour l'utilisation du Rusitec (Blanchart et al, 1989).

La mise en route du fermenteur est effectuée en introduisant dans chaque réacteur un inoculum liquide constitué de 1,25 I de jus de rumen et 1,25 I d'un mélange de solution saline (tableau I) et d'eau (60/40). Le jus de rumen est obtenu à partir de contenu total de rumen qui est prélevé sur au moins 3 moutons munis d'une canule permanente du rumen. Les moutons reçoivent une ration quotidienne constituée de 
Tableau I. Composition de la solution saline.

Macro-éléments (g/l)

Oligo-éléments (mg/l)

$\begin{array}{lllr}\mathrm{NaHCO}_{3} & 9,240 & & \\ \mathrm{Na}_{2} \mathrm{HPO}_{4}, 12 \mathrm{H}_{2} \mathrm{O} & 7,125 & \mathrm{FeSO}_{4}, 7 \mathrm{H}_{2} \mathrm{O} & 75,00 \\ \mathrm{NaCl} & 0,470 & \mathrm{MnSO}_{4}, \mathrm{H}_{2} \mathrm{O} & 4,00 \\ \mathrm{KCl} & 0,450 & \mathrm{CoCl}_{2}, 6 \mathrm{H}_{2} \mathrm{O} & 3,54 \\ \mathrm{MgCl}_{2}, 6 \mathrm{H}_{2} \mathrm{O} & 0,100 & \mathrm{CuSO}_{4}, 5 \mathrm{H}_{2} \mathrm{O} & 2,00 \\ \mathrm{CaCl}_{2}, 2 \mathrm{H}_{2} \mathrm{O} & 0,073 & \mathrm{ZnSO}_{4}, 7 \mathrm{H}_{2} \mathrm{O} & 0,10 \\ \mathrm{Na}_{2} \mathrm{SO}_{4} & 0,068 & & \end{array}$

$500 \mathrm{~g}$ de luzerne déshydratée et de foin de prairie permanente à volonté (ingestion approximative : $600 \mathrm{~g} / \mathrm{j}$ ). Ce contenu de rumen est pressé a travers 3 couches de gaze, au plus tard 30 min après le prélèvement, juste avant le remplissage des réacteurs.
Les aliments (tableau II) sont utilisés, dans la mesure du possible, sans traitement préalable. Les aliments déshydratés sont utilisés en l'état. Les aliments secs non moulus sont broyés à 1,7 $\mathrm{mm}$. Les aliments humides sont lyophilisés puis broyés à $1,7 \mathrm{~mm}$.

Tableau II. Composition chimique des aliments.

\begin{tabular}{|c|c|c|c|c|c|c|c|c|}
\hline \multirow{4}{*}{ Aliments } & \multirow{2}{*}{\multicolumn{3}{|c|}{$\begin{array}{l}\text { Composition } \\
\text { de la } \\
\text { matière sèche } \\
\text { (\%) }\end{array}$}} & \multicolumn{5}{|c|}{ Fractions azotées } \\
\hline & & & & \multicolumn{3}{|c|}{ en $\%$ de $N_{T}$} & \multicolumn{2}{|c|}{ en $\%$} \\
\hline & $N_{T} \times 6,25$ & $N D F$ & $A D F$ & $N_{S}$ & $N D I N$ & $A D I N$ & $N_{S}$ & $A D I N$ \\
\hline & & & & & & & NDSN & NDIN \\
\hline Tourteau de soja & 51,5 & 21,3 & 12,5 & 6,2 & 15,4 & 1,8 & 7,3 & 11,7 \\
\hline Tourteau de colza & 36,1 & 27,4 & 18,2 & 15,4 & 30,5 & 6,2 & 22,2 & 20,3 \\
\hline Tourteau de tournesol & 34,9 & 43,7 & 31,6 & 25,1 & 9,1 & 4,0 & 27,6 & 44,0 \\
\hline Graine de lupin & 33,8 & 35,2 & 20,1 & 29,8 & 10,5 & 1,3 & 30,7 & 12,2 \\
\hline Son de blé & 16,9 & 66,0 & 14,8 & 27,5 & 45,5 & 3,2 & 50,5 & 7,0 \\
\hline Luzerne déshydratée 1 & 20,8 & 44,5 & 27,5 & 25,5 & 51,2 & 11,6 & 52,3 & 22,7 \\
\hline Luzerne déshydratée 2 & 16,7 & 51,8 & 32,2 & 13,9 & 44,3 & 9,2 & 23,7 & 20,8 \\
\hline Foin de luzerne & 18,8 & 48,4 & 36,5 & 26,5 & 22,6 & 15,1 & 34,2 & 66,8 \\
\hline Ensilage de maïs & 8,0 & 41,6 & 24,0 & 50,0 & 28,7 & 10,0 & 70,1 & 34,8 \\
\hline Maïs déshydraté & 9,0 & 55,6 & 24,7 & 14,6 & 40,3 & 17,4 & 24,4 & 43,2 \\
\hline Résidu de pressage de raisin & 15,0 & 66,7 & 55,8 & 5,2 & 69,8 & 43,2 & 17,2 & 61,9 \\
\hline
\end{tabular}

$\mathrm{N}_{\mathrm{T}}$ : Azote total; NDF : Neutral detergent fiber, estimation des parois totales; ADF : Acid detergent fiber, estimation de la lignocellulose; $N_{S}$ : Azote soluble dans une solution saline; NDSN : Azote soluble dans le détergent neutre de Van Soest; NDIN : Azote insoluble dans le détergent neutre de Van Soest; ADIN : Azote insoluble dans le détergent acide de Van Soest. 
Dès la mise en route du fermenteur, la solution saline est infusée en continu à raison de $1500 \mathrm{ml} / \mathrm{j}$. Le réglage du niveau de liquide dans les réacteurs déterminant un volume utile de $2500 \mathrm{ml}$, ce débit correspond à un taux de renouvellement de la phase liquide de $0,6 / \mathrm{j}$.

En fonctionnement habituel, 4 sachets contenant l'aliment à tester sont présents dans chaque réacteur. Ces sachets sont remplacés par paire tous les jours. Le temps de séjour normal d'un sachet est donc de $48 \mathrm{~h}$.

Dans l'étude de la cinétique de dégradation, les sachets devant séjourner moins de $48 \mathrm{~h}$ ( 2 , $4,8,12$ et 24 h) dans le réacteur sont retirés après la durée de fermentation désirée. lls sont remplacés par des sachets neufs qui entrent dans le cycle de routine. Si ce protocole se renouvelait à une fréquence trop élevée il risquerait de provoquer une augmentation progressive de la concentration en produits terminaux dans le milieu de fermentation, consécutivement à l'accroissement de la quantité d'aliment mise à fermenter chaque jour. Pour pallier cet inconvénient, le rythme de routine est repris pendant au moins 2 jours entre 2 mesures. La veille de chaque mesure, un contrôle des conditions de milieu dans les réacteurs est pratiqué de manière à vérifier que ces conditions sont semblables à celles observées en routine.

Pour chaque calcul de disparition, du contenu de réacteur est prélevé en début et en fin de période. Ces échantillons sont utilisés pour le dosage de l'azote ammoniacal par potentiométrie.

Deux réacteurs sont employés pour chaque aliment et le nombre de répétitions pour chaque durée est fixé de telle manière que la quantité de résidu, obtenue après regroupement, soit suffisante pour permettre l'analyse (2 à 10 sachets par réacteur).

À leur sortie des réacteurs, les sachets sont rincés 2 fois avec une solution saline à $40^{\circ} \mathrm{C}$. Le liquide de rinçage est réintroduit dans le réacteur de manière à faciliter le maintien de la population microbienne par un réensemencement journalier. Les sachets sont ensuite lavés en machine à l'eau courante froide en 2 cycles de lavage de 6 min. Á l'issue de ce lavage, l'eau exprimée des sachets doit être parfaitement limpide et incolore. Les sachets subissent alors un présèchage d'au moins $10 \mathrm{~h}$ à température ambiante (de manière à éliminer la plus grande partie de l'eau résiduelle) avant d'être complète- ment séchés $24 \mathrm{~h}$ à $80^{\circ} \mathrm{C}$. Cette pratique permet de réduire les effets du chauffage sur la composition chimique des résidus.

Les aliments et les résidus de fermentation sont analysés afin de permettre le calcul, pour chacun des aliments et chacune des durées de fermentation, des quantités disparues de matière sèche, de matière organique, de fraction NDF (Van Soest et Wine, 1967) d'azote total et d'azote insoluble dans le détergent neutre de Van Soest (NDIN). Sur les aliments sont également mesurées les teneurs en ADF (Van Soest, 1963) et en azote insoluble dans le détergent acide de Van Soest (ADIN).

Pour chaque aliment et chaque composant, l'équation de la cinétique de dégradation est calculée à l'aide de la procédure de régression non linéaire NLIN (SAS, 1987) ou, à défaut, par régression linéaire.

\section{RÉSULTATS}

La cinétique de dégradation de 11 aljments a été étudiée (tableau III). Les durées de 4, 12, 24 et 48 h ont été systématiquement retenues. Pour améliorer la description de la cinétique de dégradation, les durées de $2 \mathrm{~h}$ (4 aliments) et de $8 \mathrm{~h}$ (5 aliments) ont été incluses dans l'étude, dans la mesure des possibilités.

D'une manière générale, la matière sèche et ses constituants disparaissent de moins en moins vite avec la durée de fermentation. Entre 24 et $48 \mathrm{~h}$ de fermentation, la disparition varie peu et les écarts rapportés à la différence de durée (24 h), correspondant aux vitesses de disparition au cours de cette période, deviennent très faibles $(0,1$ à $0,5 \% / h$ pour la matière sèche).

Quelle que soit la durée d'incubation, la disparition de la matière sèche est légèrement supérieure à celle de la matière organique. La fraction NDF présente des taux de disparition nettement inférieurs. La disparition de l'azote total est, en revanche, plus forte que celle de la matière organi- 
Tableau III. Evvolution en fonction du temps de séjour en Rusitec de la disparition des principaux composants des aliments.

(a) matière sèche

\begin{tabular}{|c|c|c|c|c|c|c|c|c|c|c|c|}
\hline \multirow{2}{*}{ Aliment } & \multicolumn{6}{|c|}{ Disparition (D) } & \multicolumn{3}{|c|}{$D=a+b\left(1-\theta^{-c t}\right)$} & \multicolumn{2}{|c|}{$D=a t+b$} \\
\hline & 2 & 4 & 8 & 12 & 24 & 48 & $a$ & $b$ & $c$ & $a$ & $b$ \\
\hline Tourteau de soja & ND & 47,0 & ND & 73,8 & 82,5 & 95,1 & 28,8 & 65,8 & 8,5 & & \\
\hline Tourteau de colza & 48,6 & 52,2 & 62,5 & 65,0 & 75,2 & 81,3 & 43,2 & 39,0 & 7,3 & & \\
\hline Tourteau de tournesol & ND & 50,4 & ND & 63,2 & 69,2 & 71,5 & 38,2 & 33,4 & 11,4 & & \\
\hline Graine de lupin & 56,0 & 59,1 & 62,1 & 72,4 & 89,7 & 97,0 & 49,2 & 54,8 & 4,6 & & \\
\hline Son de blé & ND & 42,7 & ND & 58,4 & 68,2 & 71,2 & 28,3 & 43,4 & 10,0 & & \\
\hline Luzerne déshydratée 1 & ND & 48,9 & 55,5 & 64,8 & 75,8 & 81,7 & 35,9 & 47,3 & 7,5 & & \\
\hline Luzerne déshydratée 2 & 39,2 & 42,9 & 46,7 & 50,1 & 63,8 & 72,4 & 35,9 & 44,6 & 3,7 & & \\
\hline Foin de luzerne & ND & 35,6 & ND & 48,6 & 51,0 & 61,4 & 30,6 & 32,7 & 5,1 & & \\
\hline Ensilage de maïs & ND & 54,3 & ND & 55,4 & 56,6 & 62,0 & & & & 0,18 & 53,2 \\
\hline Maïs deshydraté & 45,6 & 49,6 & 50,2 & 51,4 & 52,9 & 57,7 & 46,3 & 13,5 & 3,5 & & \\
\hline Résidu de pressage & ND & 19,8 & ND & 20,7 & 23,0 & 30,9 & & & & 0,26 & 17,9 \\
\hline
\end{tabular}

(b) Matière organique

\begin{tabular}{|c|c|c|c|c|c|c|c|c|c|c|c|}
\hline \multirow{2}{*}{ Aliment } & \multicolumn{6}{|c|}{ Disparition (D) } & \multicolumn{3}{|c|}{$D=a+b\left(1-e^{-c t}\right)$} & \multicolumn{2}{|c|}{$D=a t+b$} \\
\hline & 2 & 4 & 8 & 12 & 24 & 48 & a & $b$ & $c$ & $a$ & $b$ \\
\hline Tourteau de soja & ND & 44,7 & ND & 72,3 & 81,5 & 94,9 & 26,4 & 68,2 & 8,3 & & \\
\hline Tourteau de colza & 48,4 & 51,8 & 62,1 & 64,3 & 74,8 & 81,1 & 43,2 & 39,1 & 7,0 & & \\
\hline Tourteau de tournesol & ND & 48,1 & ND & 60,9 & 67,1 & 69,5 & 36,0 & 33,5 & 11,2 & & \\
\hline Graine de lupin & 55,3 & 58,3 & 61,3 & 71,9 & 89,5 & 97,1 & 48,4 & 56,1 & 4,6 & & \\
\hline Son de blé & ND & 41,1 & ND & 55,7 & 66,3 & 69,4 & 27,9 & 42,3 & 9,2 & & \\
\hline Luzerne déshydratée 1 & ND & 45,9 & 52,9 & 62,6 & 74,0 & 80,5 & 32,5 & 49,7 & 7,4 & & \\
\hline Luzerne déshydratée 2 & 35,1 & 39,1 & 43,6 & 47,4 & 61,2 & 70,8 & 31,7 & 47,2 & 3,8 & & \\
\hline Foin de luzerne & ND & 45,0 & ND & 58,8 & 60,4 & 69,0 & 37,9 & 30,7 & 7,5 & & \\
\hline Ensilage de maïs & ND & 60,8 & ND & 66,2 & 73,2 & 73,9 & 55,1 & 19,8 & 8,0 & & \\
\hline Maïs déshydraté & 45,0 & 48,0 & 48,4 & 51,5 & 51,4 & 56,3 & 45,1 & 12,4 & 4,2 & & \\
\hline Résidu de pressage & ND & 19,1 & ND & 20,3 & 22,3 & 30,6 & & & & 0,27 & 17,2 \\
\hline
\end{tabular}

que. L'azote insoluble dans le détergent neutre disparaît généralement plus que l'ensemble de la fraction NDF.

La cinétique de teneur en ammoniac du milieu de fermentation est présentée dans le tableau IV. La concentration de base, évaluée par la teneur mesurée au temps 0 (c'est-à-dire immédiatement après le renouvellement des sachets), est généralement supérieure à $5 \mathrm{mg} / 100 \mathrm{ml}$, valeur souvent considérée comme seuil pour une activité microbienne normale. Seuls des aliments pauvres en azote (maïs déshydraté ou ensilé) ou dont l'azote est très fai- 
(c) Fraction NDF

\begin{tabular}{|c|c|c|c|c|c|c|c|c|c|c|c|}
\hline \multirow{2}{*}{ Aliment } & \multicolumn{6}{|c|}{ Disparition (D) } & \multicolumn{3}{|c|}{$D=a+b\left(1-e^{-c t}\right)$} & \multicolumn{2}{|c|}{$D=a t+b$} \\
\hline & 2 & 4 & 8 & 12 & 24 & 48 & $a$ & $b$ & $c$ & $a$ & $b$ \\
\hline Tourteau de soja & ND & 12,6 & ND & 44,3 & 70,0 & 86,2 & $-11,1$ & 101,6 & 6,6 & & \\
\hline Tourteau de colza & 1,6 & 7,5 & 14,8 & 21,3 & 37,4 & 47,8 & $-3,8$ & 56,8 & 5,1 & & \\
\hline Tourteau de tournesol & ND & 8,6 & ND & 25,2 & 33,5 & 37,0 & $-6,6$ & 43,7 & 10,7 & & \\
\hline Graine de lupin & 23,4 & 33,3 & 40,7 & 43,7 & 76,9 & 93,0 & 17,4 & 93,3 & 3,6 & & \\
\hline Son de blé & ND & 27,6 & ND & 43,2 & 53,6 & 58,2 & 14,5 & 44,5 & 8,7 & & \\
\hline Luzerne déshydratée 1 & ND & 26,6 & 24,8 & 36,6 & 54,8 & 65,0 & 13,7 & 61,4 & 4,0 & & \\
\hline Luzerne déshydratée 2 & 14,5 & 17,9 & 19,7 & 24,3 & 40,8 & 54,7 & 11,0 & 71,3 & 2,0 & & \\
\hline Foin de luzerne & ND & (1) & ND & 13,2 & 32,4 & 47,5 & $-23,9$ & 76,9 & 5,5 & & \\
\hline Ensilage de maîs & ND & 8,4 & ND & 14,6 & 14,6 & 28,6 & & & & 0,43 & 7,1 \\
\hline Maîs déshydraté & 26,5 & 30,3 & 35,0 & 36,1 & 34,3 & 38,4 & 18,8 & 17,8 & 27,8 & & \\
\hline Résidu de pressage & ND & 13,4 & ND & 13,0 & 13,4 & 16,7 & & & & 0,08 & 12,3 \\
\hline
\end{tabular}

(1) Valeur négative

(d) Azote total

\begin{tabular}{|c|c|c|c|c|c|c|c|c|c|c|c|}
\hline \multirow{2}{*}{ Aliment } & \multicolumn{6}{|c|}{ Disparition (D) } & \multicolumn{3}{|c|}{$\mathrm{D}=\mathrm{a}+\mathrm{b}\left(1-\theta^{-c t}\right)$} & \multicolumn{2}{|c|}{$D=a t+b$} \\
\hline & 2 & 4 & 8 & 12 & 24 & 48 & $a$ & $b$ & $c$ & $a$ & $b$ \\
\hline Tourteau de soja & ND & 44,8 & ND & 68,8 & 78,8 & 95,2 & 32,0 & 65,9 & 5,9 & & \\
\hline Tourteau de colza & 56,8 & 59,8 & 69,6 & 75,9 & 85,9 & 91,7 & 49,9 & 43,0 & 7,6 & & \\
\hline Tourteau de tournesol & ND & 80,0 & ND & 88,2 & 95,2 & 96,5 & 72,2 & 25,0 & 9,1 & & \\
\hline Graine de lupin & 80,9 & 83,8 & 85,8 & 92,7 & 97,1 & 99,3 & 77,2 & 22,9 & 7,9 & & \\
\hline Son de blé & ND & 56,7 & ND & 81,6 & 93,4 & 94,1 & 28,7 & 66,2 & 13,7 & & \\
\hline Luzerne déshydratée 1 & ND & 45,7 & 51,3 & 63,2 & 80,1 & 89,7 & 30,8 & 63,4 & 5,8 & & \\
\hline Luzerne déshydratée 2 & 39,5 & 42,7 & 48,7 & 50,8 & 71,1 & 85,7 & 35,1 & 72,3 & 2,6 & & \\
\hline Foin de luzerne & ND & 56,1 & ND & 67,3 & 73,6 & 81,6 & 49,8 & 33,9 & 5,5 & & \\
\hline Ensilage de maïs & ND & 84,4 & ND & 83,0 & 83,6 & 85,0 & & & & 0,02 & 83,6 \\
\hline Maïs déshydraté & 50,6 & 53,3 & 50,4 & 51,4 & 54,4 & 58,0 & & & & 0,14 & 51,0 \\
\hline Résidu de pressage & ND & 21,6 & ND & 24,0 & 22,8 & 33,0 & & & & 0,25 & 19,9 \\
\hline
\end{tabular}

blement dégradable (résidu de pressage du raisin) induisent des teneurs en ammoniac faibles. Le manque de disponibilité en azote pour les bactéries est probablement à la fois cause et conséquence du faible taux de dégradation de ces aliments. La variation dans le temps de la teneur en ammoniac du milieu de fermentation est généralement assez faible. Cette teneur peut cependant prendre une valeur maximale dans le cas des tourteaux.

\section{DISCUSSION}

Les cinétiques de disparition de la matière sèche et de ses constituants sont, le plus souvent, décrites par le modèle exponen- 
Tableau III (suite)

(e) Azote insoluble dans le détergent neutre (NDIN)

\begin{tabular}{|c|c|c|c|c|c|c|c|c|c|c|c|}
\hline \multirow{2}{*}{ Aliment } & \multicolumn{6}{|c|}{ Disparition (D) } & \multicolumn{3}{|c|}{$\mathrm{D}=\mathrm{a}+\mathrm{b}\left(1-e^{-\mathrm{ct}}\right)$} & \multicolumn{2}{|c|}{$D=a t+b$} \\
\hline & 2 & 4 & 8 & 12 & 24 & 48 & $a$ & $b$ & $c$ & a & $b$ \\
\hline Tourteau de soja & ND & 9,8 & ND & 21,3 & 69,2 & 82,8 & $-12,0$ & 112,3 & 4,2 & & \\
\hline Tourteau de coiza & 41,5 & 49,5 & 55,5 & 60,5 & 69,2 & 82,2 & 39,5 & 46,6 & 4,8 & & \\
\hline Tourteau de tournesol & ND & 11,9 & ND & 48,6 & 71,7 & 77,3 & $-22,9$ & 101,6 & 10,4 & & \\
\hline Graine de lupin & 19,6 & 33,3 & 50,1 & 58,2 & 82,6 & 91,5 & 8,1 & 85,5 & 8,0 & & \\
\hline Son de blé & ND & 49,2 & ND & 80,6 & 90,2 & 91,1 & 7,5 & 83,8 & 17,2 & & \\
\hline Luzerne déshydratée 1 & ND & 28,5 & 23,8 & 45,2 & 70,0 & 84,9 & 8,5 & 90,0 & 4,2 & & \\
\hline Luzerne déshydratée 2 & 2,6 & 31,7 & 36,5 & 41,2 & 56,1 & 80,0 & 5,1 & 77,9 & 5,5 & & \\
\hline Foin de luzerne & ND & 27,8 & ND & 32,9 & 34,9 & 57,3 & & & & 0,66 & 23,7 \\
\hline Ensilage de maïs & ND & 33,8 & ND & 41,3 & 46,2 & 48,5 & 27,6 & 21,3 & 8,7 & & \\
\hline Maïs déshydraté & 12,9 & 34,9 & 22,9 & 33,2 & 41,4 & 47,8 & 16,0 & 33,7 & 5,8 & & \\
\hline Résidu de pressage & ND & 9,4 & ND & 11,0 & 0,1 & 14,5 & & & & 0,09 & 6,8 \\
\hline
\end{tabular}

tiel négatif proposé par Ørskov et McDonald (1979) $\left[p=a+b\left(1-e^{-c t}\right)\right]$. Ce modèle peut être caractérisé par 3 paramètres :

- la dégradabilité maximale, correspondant à l'ordonnée de l'asymptote de la courbe et représentée par la somme des paramètres $a$ et $b$ de l'équation,
- la dégradabilité instantanée, correspondant à l'ordonnée à l'origine de la courbe et représentée par le paramètre a de l'équation,

- la vitesse de dégradation de la fraction lentement dégradable qui est représentée par le paramètre $c$ de l'équation.

Tableau IV. Évolution de la concentration en ammoniac $\left(\mathrm{mg} \mathrm{NH}_{3} / 100 \mathrm{ml}\right)$ des contenus des réacteurs au cours de la fermentation de différents aliments en Rusitec.

\begin{tabular}{lcccc}
\hline \multirow{2}{*}{ Aliment } & \multicolumn{4}{c}{ Durées de fermentation $(h)$} \\
\cline { 2 - 5 } & 0 & 4 & 12 & 24 \\
\hline & & & & \\
Tourteau de soja & 44,1 & 47,8 & 94,7 & 67,9 \\
Tourteau de colza & 37,2 & 22,8 & 44,2 & 47,1 \\
Tourteau de tournesol & 28,9 & 41,9 & 30,9 & 31,7 \\
Son de blé & 17,6 & 21,7 & 20,0 & 19,5 \\
Graines de lupin & 54,9 & 58,0 & 60,6 & 47,3 \\
Luzerne déshydratée 1 & 14,4 & 16,0 & 13,6 & 15,3 \\
Luzerne déshydratée 2 & 5,9 & 7,7 & 5,5 & 4,0 \\
Foin de luzerne & 11,4 & 44,6 & 12,5 & 20,3 \\
Ensilage de maïs & 1,5 & 4,8 & 1,9 & 1,2 \\
Maïs déshydraté & 0,11 & 0,12 & 0,05 & 0,12 \\
Résidu de pressage & 0,17 & 0,23 & 0,07 & 0,06 \\
\hline
\end{tabular}


Tableau V. Valeurs des paramètres caractérisant les courbes moyennes de cinétique de dégradation de l'azote pour différents aliments (valeurs calculées à partir de $n$ données bibliographiques obtenues in situ par la méthode des sachets).

\begin{tabular}{|c|c|c|c|c|c|}
\hline Aliments & $n$ & $a$ & $b$ & $c$ & Références \\
\hline Tourteau de soja & 26 & 14,0 & 84,7 & 8,9 & $\begin{array}{l}a, c, d, f, g, h, i, j, k, l, m, n, o, \\
q, r, s, t, u, v, w, x\end{array}$ \\
\hline Tourteau de colza & 12 & 25,4 & 66,3 & 10,0 & $a, e, h, k, o, q$ \\
\hline Tourteau de tournesol & 4 & 30,5 & 67,4 & 12,9 & $e, g$ \\
\hline Lupin & 1 & 74,3 & 21,9 & 34,3 & $e^{3}$ \\
\hline Son de blé & 1 & 50,0 & 42,0 & 16,1 & a \\
\hline Luzerne déshydratée & 4 & 35,9 & 52,7 & 4,9 & $a, h, k, p$ \\
\hline Foin de luzerne & 11 & 39,5 & 48,2 & 8,7 & $b, k$ \\
\hline
\end{tabular}

a : Abe, 1983; b : Alvir et al, 1988; $c$ : Armentano et al, 1986; d : Erdman et al, 1987; e : Freer et Dove, 1984; $f:$ Firkins ot al, 1984; $\mathrm{g}$ : Ganev et al, 1979; $\mathrm{h}$ : Ha et Kennelly, 1984; $\mathrm{i}$ : Hennessy et al, 1983; J : Kayouli et al, 1983; $\mathrm{k}$ : Kennelly et al, 1986; I : Kovacik et al, 1986; $\mathrm{m}$ : Kristensen et al, 1982; $\mathrm{n}$ : Lindberg, 1981: o : Lindberg et Varvikko, 1982; $p$ : Mathers et Miller, 1981; $q$ : Mir et al, 1984; $r$ : Orskov et al,. 1983; s : Ørskov et McDonald, 1979; $t$ : Stern et al, 1983; $u$ : Stern et al, 1985; $v$ : Ushida et Jouany, 1985; $w$ : Weakley et al, 1983; $x$ : Zerbini et Polan, 1985.

Les équations représentant la cinétique de dégradation de l'azote (tableau III) sont proches des équations moyennes établies à partir des mesures pratiquées par la méthode des sachets in situ par différents auteurs pour des aliments semblables (tableau V). En particulier, la localisation de l'asymptote, c'est-à-dire la valeur de $a+b$ calculée à partir de nos résultats, est très peu différente de celle calculée d'après les résultats fournis par la bibliographie.

La différence la plus marquée concerne la localisation de l'ordonnée à l'origine, c'est-à-dire la valeur de a. Le paramètre a est souvent plus grand dans nos résultats in vitro que dans les résultats de la bibliographie obtenus in situ. Cette différence traduit une disparition plus forte de l'azote dans nos essais in vitro pour les faibles durées de fermentation. Elle peut aussi être due à une certaine contamination, par des fines particules solides, des résidus des sachets placés in situ. L'écart s'atté- nue progressivement, la vitesse de disparition de la fraction lentement dégradable étant généralement un peu plus faible dans nos essais in vitro.

Les cas du résidu de pressage de raisin, de l'ensilage de maïs et du maïs déshydraté se caractérisent par une très faible vitesse de disparition de la fraction lentement dégradable. Pour ces aliments, le modèle exponentiel n'est pas satisfaisant dans nos conditions de mesure et il semble opportun de lui préférer le modèle linéaire voire, dans le cas de l'ensilage de maïs, une valeur constante.

\section{Dégradabilité maximale}

Avec les aliments pour lesquels il a été possible d'utiliser le modèle exponentiel, la dégradabilité maximale de l'azote, estimée par l'ordonnée à l'origine de l'asymptote 
a) Tourteau de soja

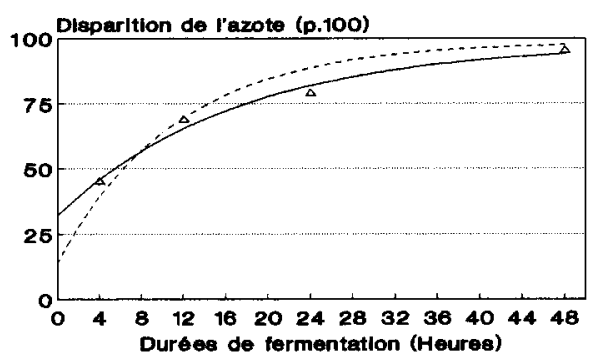

c) Tourteau de tournesol

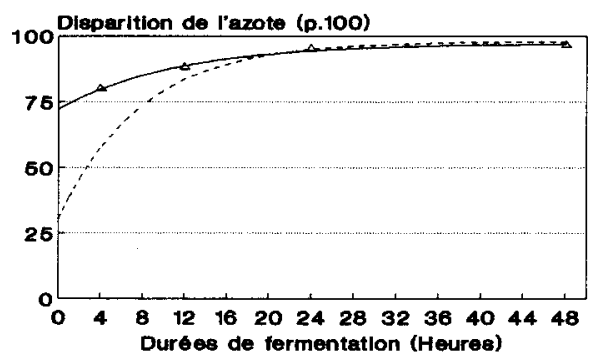

e) Son de ble

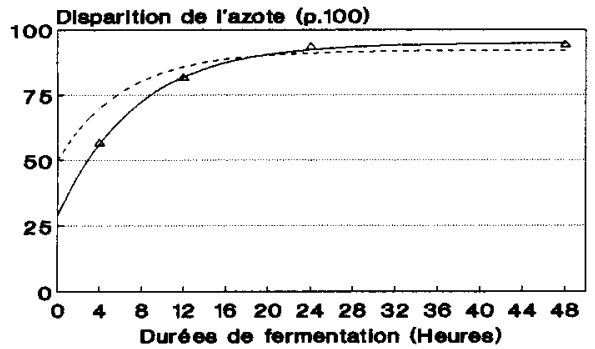

b) Tourteau de colza

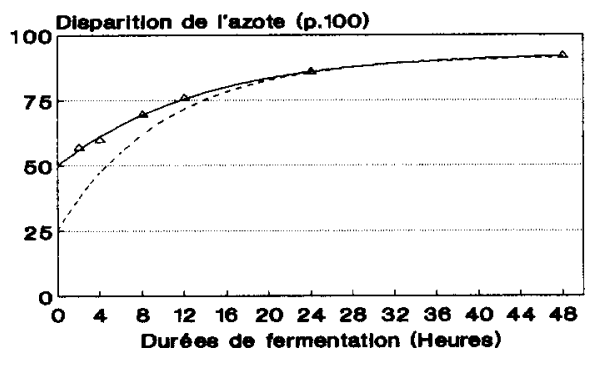

d) Graine de lupin

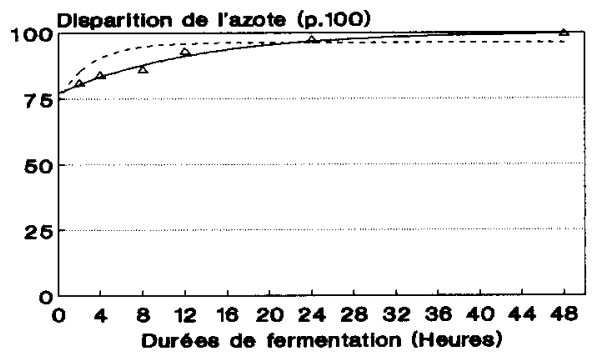

f)Luzerne deshydratee

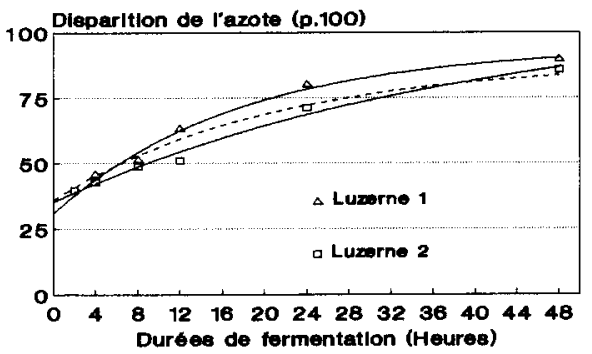

g)Foin de luzerne

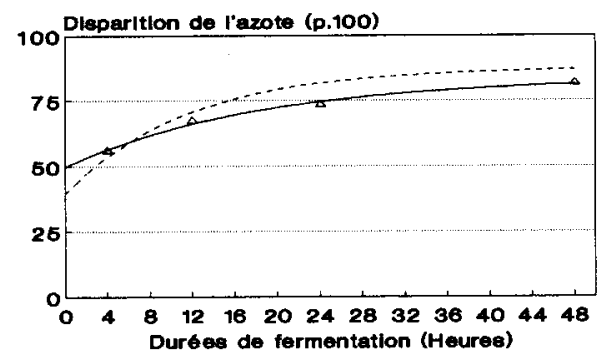

Fig 1. Cinétiques de disparition de l'azote total pour différents aliments. Comparaison entre les résultats obtenus en Rusitec $(-\Delta)$ et les courbes établies à partir des données bibliographiques obtenues in situ et présentées dans le tableau IV (-----). 
$\left[(a+b)_{N T}\right]$, est assez étroitement liée à la disparition en $48 \mathrm{~h}\left(\mathrm{D}_{\mathrm{NT} 48}\right)$ :

$$
\begin{gathered}
(a+b)_{N T}=0,599 \pm 0,275 D_{N T 48}+ \\
40,2 \pm 4,3 \quad r=0,664 ; n=8
\end{gathered}
$$

Il est donc possible d'envisager la mesure de ce taux de disparition pour prévoir la dégradabilité maximale. Ces observations vont dans le même sens que celles d'Ørskov et McDonald (1979) selon lesquels, pour la plupart des aliments et régimes, le taux de disparition de l'azote pour une durée d'incubation de $24 \mathrm{~h}$ peut être une estimation satisfaisante de sa dégradabilité maximale.

La dégradabilité maximale de l'azote est aussi fortement liée à la composition des aliments et en particulier à la proportion de l'azote qui est insoluble dans le détergent acide de $\operatorname{Van}$ Soest $\left(A D I N / N_{T}\right)$ :

$$
\begin{gathered}
(a+b)_{N T}=-0,760 \pm 0,303 \text { ADIN/N } \\
+100,1 \pm 4,0 \quad r=-0,716 ; n=8
\end{gathered}
$$

Cette relation met en évidence l'effet de la protection des matières azotées par les constituants de l'ADF contre les fermentations, exprimé par la résistance de l'azote à la solubilisation dans le détergent acide de Van Soest. Compte tenu de la valeur prise par le terme constant (non différente de 100), elle permet de considérer la fraction indégradable comme proportionnelle à la fraction $A D I N$. II paraît ainsi possible d'envisager la mesure de la fraction ADIN pour prévoir la dégradabilité maximale.

L'opportunité de la prise en compte de la fraction ADIN pour exprimer la résistance de l'azote aux fermentations dans le rumen est confirmée par la relation entre la dégradabilité maximale de la fraction NDIN $\left[(a+b)_{N D I N}\right]$, estimation de la dégradabilité maximum des matières azotées pariétales, et la part de ADIN dans NDIN :

$$
\begin{gathered}
(a+b)_{\text {NDIN }}=-1,073 \pm 0,348 \text { ADIN/NDIN + } \\
106,9 \pm 13,4 \quad r=-0,759 ; n=9
\end{gathered}
$$

Cette relation traduit l'effet de protection que des liaisons entre les protéines et la fraction ligno-cellulosique exercent sur la dégradation de ces protéines.

Les protéines liées à la fraction lignocellulosique des parois se trouvent protégées des actions microbiennes. Leur disparition, et par conséquent, celle de l'azote total est d'autant plus réduite que cette protection est plus développée. Krishnamoorthy et al (1982b) considèrent que la fraction azotée insoluble dans le détergent acide est absolument indégradable dans le rumen. II n'est donc pas surprenant que la part de l'azote qui résiste aux fermentations soit proportionnelle à la part de la fraction ADIN dans l'azote total. Plusieurs auteurs ont mentionné la diminution de la dégradabilité de l'azote qui accompagne l'augmentation de la proportion de la fraction ADIN consécutive à un traitement à la chaleur (Lindberg et al, 1982; Pena et al, 1986). Cet effet du blocage de l'azote semble ici pouvoir être généralisé, indépendamment des traitements thermiques. II ne paraît cependant pas possible d'attribuer une signification nutritionnelle simple aux coefficients de régression des équations.

\section{Dégradabilité instantanée}

Le paramètre a du modèle exponentiel, estimation de la dégradation instantanée de l'azote, n'est étroitement lié à aucun des critères de composition chimique étudiés. Les meilleures relations sont obtenues avec la solubilité de l'azote dans la solution saline (NS/NT) et avec la solubilité dans le détergent neutre de Van Soest (NDIN/NT): $a_{N T}=0,991 \pm 0,837 N_{S} / N_{T}+25,9 \pm 18,5$ $r=0,435 ; n=8$ 
$a_{N T}=-0,872 \pm 0,296 \mathrm{NDIN} / \mathrm{N}_{\mathrm{T}}+71,9$ $\pm 13,1$

$r=-0,769 ; n=8$

La difficulté pour expliquer le paramètre $a$ avec des critères de composition chimique tient probablement au fait que, dans les équations décrivant la cinétique de disparition de l'azote total, $a$ et $b$ ne sont pas indépendants et que, pour des formes de courbes semblables, a peut prendre des valeurs très différentes. De plus, le modèle exponentiel utilisé ne tient pas compte d'un éventuel temps de latence appliqué à la dégradation de la fraction lentement dégradable, en particulier si ce temps de latence est inférieur à la plus courte des durées d'incubation testées.

\section{Vitesse de dégradation}

Dans nos essais, la vitesse de dégradation de la fraction lentement dégradable de l'azote (c) est négativement corrélée au rapport ADF/NDF

$$
\begin{aligned}
& C_{N T}=-0,138 \pm 0,059 \text { ADF/NDF }+15,5 \\
& \pm 2,5 \\
& r=-0,693 ; n=8
\end{aligned}
$$

Cette relation caractérise l'effet de frein ou de protection de la fraction lignocellulosique sur l'attaque des matières azotées pariétales, et, par conséquent, sur la dégradation de l'azote lentement dégradable.

Cette protection est probablement plus forte lorsque les conditions de fermentation sont moins favorables aux activités cellulolytiques. Cette observation expliquerait, au moins partiellement, la diminution de la dégradation de l'azote des fourrages qui peut survenir lorsqu'on ajoute du concentré (céréale) dans la ration (Loerch et al, 1983; Weakley et al, 1983; Zinn et Owens, 1983).

\section{CONCLUSION}

Les matières azotées végétales sont en partie résistantes à la solubilisation dans les détergents de Van Soest. Cette résistance peut être due à leur localisation dans les parois cellulaires ou à des liaisons chimiques que certains traitements technologiques ont pu occasionner. Elles sont un témoin de leur résistance aux activités microbiennes dans le rumen. Ainsi la solubilité de la matière organique, et spécialement de l'azote, dans les détergents de Van Soest serait un moyen de prédire les paramètres de l'équation décrivant la cinétique de dégradation de l'azote.

\section{RÉFÉRENCES}

Abe $M(1983)$ Degradation properties and the susceptibility to degradation of feed protein in the rumen of cows. In: $4^{\theta}$ Symp Int Métabolisme et nutrition azotés, INRA, Publ, Versailles, 223-226

Alvir MR, Gonzalez J, Argamentaria A (1988) Relations entre la composition chimique des foins de luzerne et la dégradabilité in sacco de leurs matières azotées. Reprod Nutr Dev $28,1,169-170$

Armentano LE, Herrington TA, Polan CE, Moe AJ, Herbein JH, Umstadt $P$ (1986) Ruminal degradation of dried brewers grains, wet brewers grains and soybean meal. J Dairy Sci 69, 2124-2133

Blanchart G, Vignon B (1984) Adaptation d'un fermenteur de type Rusitec. Cah Tech INRA $7,1-10$

Blanchart G, Durand M, Barry JL, Bouiller-Oudot $M$, Jouany JP (1989) Intérêts et limites des fermenteurs à flux semi-continu de type Rusitec dans l'étude des fermentations du rumen. Ann Zootech 38, 285-314

Cotta MA, Hespell RB (1986) Proteolytic activity of the ruminal bacterium Butyrivibrio fibrisolvens. Appl Environ Microbiol 52, 51-58

Czerkawski JW, Breckenridge G (1977) Design and development of a long term rumen simu- 
lation technique (Rusitec). Br J Nutr 38, 371384

Driedger A, Hatfield EE (1972) Influence of tannins on the nutritive value of soybean meal for ruminants. J Anim Sci 34, 465-468

Erdman RA, Proctor GH, Vandersall JH (1986) Effect of rumen ammonia concentration on in situ rate and extent of digestion of feedstuffs. J Dairy Sci 69, 2312-2318

Firkins JL, Berger LL, Fahey GCJr, Merchen NR (1984) Ruminal nitrogen degradability and escape of wet and dry distillers grains and wet and dry corn gluten feed. J Dairy Sci 67, 1936-1944

Freer M, Dove H (1984) Rumen degradation of protein in sunflower meal, rapeseed meal and lupin seed placed in nylon bags. Anim Feed Sci Technol 11, 87-101

Ganev G, Orskov ER, Smart R (1979) The effect of roughage or concentrate feeding and rumen retention time on total degradation of protein in the rumen. J Agric Sci 93, 951-956

Ha JK, Kennelly JJ (1984) In situ dry matter and protein degradation of various protein sources in dairy cattle. Can $J$ Anim Sci 64, 443-452

Hartnell KA, Satter LD (1978) Effect of Masonex (hemicellulose extract) on protein degradation by rumen microorganisms in vitro and in vivo. J Anim Sci 47, 935-943

Hennessy DW, Lee GJ, Williamsson PJ (1983) Nitrogen loss from protein meals held in terylene bags in the rumen of cattle and the nutritive value of the residues. Aust $J$ Agric Res 34, 453-467

Jarrige $\mathrm{R}$ (ed) (1988) Alimentation des bovins, ovins et caprins. INRA, Paris, $476 p$

Kayouli C, Van Nevel C, Demeyer D (1983) Effet de la défaunation du rumen sur la dégradabilité des protéines du soja mesurée in sacco. $4^{\theta}$ Symp Int Métabolisme et nutrition azotés, INRA Publ, Versailles, 251-254

Kennelly JJ, Murphy JJ, de Boer G (1986) Relative value of feedstuffs as sources of rumen undegradable protein. Agric For Bull. Special issue 65th Annual Feeders' Day Report, 6164

Kovacik AM, Loerch SC, Dehority BA (1986) Effect of supplemental sodium bicarbonate on nutrient digestibilities and ruminal $\mathrm{pH}$ measured continuously. J Anim Sci 62, 226-234
Krishnamoorthy U, Muscato TV, Sniffen CJ, Van Soest PJ (1982a) Nitrogen fractions in selected feedstuffs. J Dairy Sci 65, 2, 217-225

Krishnamoorthy U, Sniffen CJ, Van Soest PJ (1982b) Nitrogen fractionation in ruminants feedstuffs for feed evaluation. Canadian Nutrition Conference for Feed Manufacturers Proceedings 95-102

Kristensen ES, Moller PD, Hvelplund T (1982) Estimation of the effective protein degradability in the rumen of cows using the nylon bag technique combined with the outflow rate. Acta Agric Scand 32, 123-127

Lindberg JE (1981) The effect of basal diet on the ruminal degradation of dry matter, nitrogenous compounds and cell walls in nylon bags. Swedish J Agric Res 11, 159-169

Lindberg JE, Varvikko $T$ (1982) The effect of bag pore size on the ruminal degradation of dry matter, nitrogenous compounds and cell walls in nylon bags. Swedish J Agric Res 12, 163-171

Lindberg JE, Soliman HS, Sannes S (1982) A study of the rumen degradability of untreated and heat-treated rape seed meal and of whole rape seed, inciuding a comparison between two nylon bag techniques. Swedish $J$ Agric Res 12, 83-88

Loerch SC, Berger LL, Gianola D, Fahey GR Jr (1983) Effect of dietary protein source and energy level on in situ nitrogen disappearance of various protein sources. $J$ Anim Sci 56, 206-216

Mahadevan S, Erfle JD, Sauer FD (1980) Degradation of soluble and insoluble proteins by Bacteroides amylophilus protease and by rumen microorganisms. J Anim Sci 50, 723728

Mathers JC, Miller EL (1981) Quantitative studies of food protein degradation and the energetic efficiency of microbial protein synthesis in the rumen of sheep given chopped lucerne and rolled barley. Br J Nutr 45,587 604

Mir Z, McLeod GK, Buchanan-Smith JG, Grieve DG, Grovum WL (1984) Methods of protecting soybean and canola proteins from degradation in the rumen. Can J Anim Sci 64, 853865

Nocek JE, Herbein JH, Polan CE (1983) Total amino acid release rates of soluble and insoluble protein fractions of concentrate feed- 
stuffs by Streptomyces griseus. J Dairy Sci 66, 1663-1667

Nugent JHA, Jones WT, Jordan DJ, Mangan JL (1983) Rates of proteolysis in the rumen of the soluble proteins casein, fraction I (18S) leaf protein, bovine serum albumin and bovine submaxillary mucoprotein. $\mathrm{Br} J$ Nutr 50 , 357-368

Ørskov ER (1982) Protein nutrition in ruminants. Acad Press, Londres, $\mathrm{p} 160$

Ørskov ER, McDonald I (1979) The estimation of protein degradability in the rumen from incubation measurements weighted according to rate of passage. J Agric Sci 92, 499-503

Ørskov ER, Hughes-Jones M, Eliman ME (1983) Studies on degradation and outflow rate of protein supplements in the rumen of sheep and cattle. Livest Prod Sci 10, 17-24

Pena F, Tagari H, Satter LD (1986) The effect of heat treatment of whole cottonseed on site and extent of protein digestion in dairy cows. $J$ Anim Sci 62, 1423-1433

SAS (1987) SAS/Stat ${ }^{T M}$ guide for personnal computers (version 6) SAS Inst Inc, Cary, NC, USA

Stern MD, Ortega ME, Satter LD (1983) Retention time in the rumen and degradation of protein supplements fed to lactating dairy cattle. J Dairy Sci66, 1264-1271

Stern MD, Santos KA, Satter LD (1985) Protein degradation in rumen and amino acid absorption in small intestine of lactating dairy cattle fed heat-treated whole soybeans. J Dairy Sci $68,45-56$
Thomas E, Trenkle A, Burroughs W (1979) Evaluation of protecting agents applied to soybean meal and fed to cattle. 1. Laboratory measurements. J Anim Sci 49, 1337-1345

Ushida K, Jouany JP (1985) Effect of rumen protozoa on rumen protein degradation in sheep. Reprod Nutr Dév 25, 1075-1081

Van Soest PJ (1963) Use of detergents in the analysis of fibrous feeds. 2. A rapid method for the determination of fiber and lignin. $J$ Assoc Off Agric Chem 46, 829-835

Van Soest PJ, Wine RH (1967) Use of detergents in the analysis of fibrous feeds. 4. Détermination of plant cell wall constituents. $J$ Assoc Off Agric Chem 50, 50-55

Weakley DC, Stern MD, Satter LD (1983) Factors affecting disappearance of feedstuffs from bags suspended in the rumen. $J$ Anim Sci 56, 493-507

Zelter SZ, Leroy F, Tissier JP (1970) Protection des protéines alimentaires contre la désamination bactérienne dans le rumen. 1. Études in vitro : comportement en milieu du rumen de quelques protéines tannées avec du tannin de châtaignier ou certains aldéhydes (formaldéhyde, glutaraldéhyde, glyoxal). Ann Biol Anim Biochim Biophys 10, 111-122

Zerbini E, Polan CE (1985) Protein sources evaluated from ruminating holstein calves. $J$ Dairy Sci 68, 1416-1424

Zinn RA, Owens FN (1983) Site of protein digestion in steers: predictability. $J$ Anim Sci 56, 707-716 